\title{
Ether Anesthetics Obstructs Touch-Induced Trigger Hair Calcium-Electrical Signals Preventing Excitation of The Venus Flytrap
}

Sönke Scherzer ( $\sim$ soenke.scherzer@uni-wuerzburg.de )

University of Würzburg

Shouguang Huang

University of Würzburg

Anda losip

University of Würzburg

Ines Fuchs

University of Würzburg

Ken Yokawa

Kitami Institute of Technology

Khaled A. S. AL-Rasheid

King Saud University

Manfred Heckmann

University of Würzburg

Rainer Hedrich

University of Würzburg

\section{Research Article}

Keywords: trigger, touch, Venus, glutamate,

Posted Date: October 26th, 2021

DOI: https://doi.org/10.21203/rs.3.rs-1000075/v1

License: (1) (1) This work is licensed under a Creative Commons Attribution 4.0 International License.

Read Full License 
Ether anesthetics prevents touch-induced trigger hair calcium-electrical signals excite the Venus flytrap

Sönke Scherzer*1, Shouguang Huang ${ }^{1}$, Anda losip ${ }^{1}$, Ines Fuchs ${ }^{1}$, Ken Yokawa ${ }^{2}$, Khaled A. S. ALRasheid ${ }^{3}$, Manfred Heckmann ${ }^{4}$, Rainer Hedrich*1

*Corrensponding authors:

soenke.scherzer@uni-wuerzburg.de

hedrich@botanik.uni-wuerzburg.de

Affiliation:

${ }^{1}$ Institute for Molecular Plant Physiology and Biophysics, University of Würzburg, Julius-vonSachs Platz 2, 97082 Würzburg, Germany.

2 School of Earth, Energy and Environmental Engineering, Faculty of Engineering, Kitami Institute of Technology, Hokkaido 090-8507, Japan

${ }^{3}$ Zoology Department, College of Science, King Saud University, P.O. Box 2455, Riyadh 11451, Saudi Arabia

4 Department of Neurophysiology, Institute of Physiology, University of Würzburg, Röntgenring 9, 97082 Würzburg, Germany 


\section{Abstract}

Plants do not have neurons but operate transmembrane ion channels and can get electrical excited by physical and chemical clues. Among them the Venus flytrap is characterized by its peculiar hapto-electric signaling. When insects collide with trigger hairs emerging the trap inner surface, the mechanical stimulus within the mechanosensory organ is translated into a calcium signal and an action potential (AP). Here we asked how the $\mathrm{Ca}^{2+}$ wave and AP is initiated in the trigger hair and how it is feed into systemic trap calcium-electrical networks. When Dionaea muscipula trigger hairs matures and develop hapto-electric excitability the mechanosensitive anion channel DmMSL10/FLYC1 and voltage dependent SKOR type Shaker $\mathrm{K}^{+}$channel are expressed in the sheering stress sensitive podium. The podium of the trigger hair is interface to the flytrap's prey capture and processing networks. In the excitable state touch stimulation of the trigger hair evokes a rise in the podium $\mathrm{Ca}^{2+}$ first and before the calcium signal together with an action potential travel all over the trap surface. In search for podium ion channels and pumps mediating touch induced $\mathrm{Ca}^{2+}$ transients, we, in mature trigger hairs firing fast $\mathrm{Ca}^{2+}$ signals and APs, found OSCA1.7 and GLR3.6 type $\mathrm{Ca}^{2+}$ channels and ACA2/10 $\mathrm{Ca}^{2+}$ pumps specifically expressed in the podium. Like trigger hair stimulation, glutamate application to the trap directly evoked a propagating $\mathrm{Ca}^{2+}$ and electrical event. Given that anesthetics affect $\mathrm{K}^{+}$channels and glutamate receptors in the animal system we exposed flytraps to an ether atmosphere. As result propagation of touch and glutamate induced $\mathrm{Ca}^{2+}$ and AP long-distance signaling got suppressed, while the trap completely recovered excitability when ether was replaced by fresh air. In line with ether targeting a calcium channel addressing a $\mathrm{Ca}^{2+}$ activated anion channel the AP amplitude declined before the electrical signal ceased completely. Ether in the mechanosensory organ did neither prevent the touch induction of a calcium signal nor this post stimulus decay. This finding indicates that ether prevents the touch activated, glr3.6 expressing base of the trigger hair to excite the capture organ. 


\section{Introduction}

Within the plant kingdom the action potential (AP) of the carnivorous Venus flytrap is most similar to the all or nothing AP in our nerve cells (Hedrich and Neher, 2018). While APs in Arabidopsis and most other plants last $>1 \mathrm{~min}$ and cannot be evoked repeatedly (Mousavi et al., 2013), the flytrap equivalent takes just about a second and can be fired a maximal frequency of $1 \mathrm{~Hz}$ (Bohm et al., 2016b). Compared to nerves with the information encoded by the frequency (number of APs per time), the flytrap system is more remote. It, however, allows Dionaea count to five APs (Bohm et al., 2016a). When a potential prey visiting the trap, attracted by color and odor, touches one of the six trigger hairs, an AP gets fired. The trigger hair is a mechano-sensitive organ that gets excited by share stress of $>3^{\circ}$ bending of $29 \mu \mathrm{N}$ force (Scherzer et al., 2019). Two AP make the trap close and imprisons the animal prey. Three and more APs trigger touch hormone jasmonate (JA) signaling and JA production. A count of 5 is required to produce digestive enzymes and transporters that are specialist to take in the animal derived nutrients (Bohm et al., 2016a).

Hapto-electric energy conversion takes place in the indentation zone of the trigger hair podium. In this zone mechano-sensitive channels of the DmMSL10 and a DmOSCA1 type are expressed (Procko et al., 2021, losip et al., 2020)). MSL10 type channels in Arabidopsis and Dionaea operate as anion channels that upon activation depolarize cells (Maksaev and Haswell, 2012), while OSCAs rather $\mathrm{Ca}^{2+}$ (Yuan et al., 2014, Murthy et al., 2018, Thor et al., 2020). To trigger excitation in the flytrap cells have to be depolarized by $20-40 \mathrm{mV}$ from the resting state (Hedrich and Fukushima, 2021). Thus, together MSL10 and OSCA(s) likely give rise to depolarization and $\mathrm{Ca}^{2+}$ influx for activation of anion channels. Anion channels in plants are the master switches in plant electrical excitation. The opening of anion channels and thus membrane electrical excitation is ultimately linked to the amplitude and kinetics of the given stimulus induced $\mathrm{Ca}^{2+}$ transient. Initial notice of a calcium-voltage-change association was made in the context of the wound/foraging response in the model plant Arabidopsis. Upon wounding the signaling molecule glutamate gets released to the extracellular space (Toyota et al., 2018), faced by ligand-binding site of GLRs (Gangwar et al., 2020, Alfieri et al., 2020). The Arabidopsis genome harbors 20 GLRs. Edgar Spalding lab in 2006 was first to demonstrate that glutamate evokes a $\mathrm{Ca}^{2+}$ signal as well as membrane depolarization which is suppressed when GLR3.3 is mutated (Qi et al., 2006). The Ted Farmer and Simon Gilroy labs have 
unequivocally shown that the electrical and $\mathrm{Ca}^{2+}$ signal, moving from the injured side in the local leaf to those wired to it via the vasculature, requires the presence GLR3.3 and 3.6 two glutamate receptors (Mousavi et al., 2013, Toyota et al., 2018).

Andrej Pavlovič and colleagues very recently have documented that ether suppresses AP firing and trap closing in Dionaea (Pavlovic et al., 2020). Cation channels responsible for the excitability of nerve cells are targets of anesthetics such as ether (Covarrubias et al., 2015, Brosnan and Pham, 2018). Key for anesthetics susceptibility are channel sites interacting with membrane lipids (Pavel et al., 2020).

Here we asked the flytrap ion channel target of ether. We found ether to suppress $\mathrm{Ca}^{2+}$ and AP stimulated trigger hairs excite capture organ. The fact that DmGLR3.6 expression was associated with flytrap excitability and the anesthetics suppressed glutamate-induced signals too, pointed to GLRs as likely ether targets.

\section{Results}

\section{Calcium-electric signal originates in trigger hair podium}

Our ecent studies have shown that the Dionaea trigger hairs can operate as sender and receiver of touch induced action potentials and $\mathrm{Ca}^{2+}$ waves (losip et al., 2020, Suda et al., 2020). How is this possible? The flytrap excitable cells rest around -140 to $-120 \mathrm{mV}$. Whenever the membrane potential is depolarized below $-100 \mathrm{mV}$ an action potential is evoked.

For technical reasons one cannot measure APs in the very trigger hair and mechanical stimulate it at the same time. However, when voltage-recording microelectrodes were inserted in to the oblong mechano-sensitive cells of the podium of a receiver trigger hair, we could receive the AP induced by touch stimulation of one the other two mechanosensory organs operating as senders (Fig. S1).

In contrast to the AP the $\mathrm{Ca}^{2+}$ signal can be recorded in the same touch stimulated organ. Two types of $\mathrm{Ca}^{2+}$ signals were picked up: i) upon sub-threshold trigger hair bending the podium cells emitting GCaMP6f fluorescence first were those of indentation zone prone to sheering stress, while ii) above-threshold stimulations lighted up the entire podium that gave rise to selfsustained $\mathrm{Ca}^{2+}$ signal that left the trigger hair and propagated along the trap surface (Fig 
1A and Supplementary Movie S1). In response to touch activation of the very trigger hair the podia of the other two touch sensitive organs of the same trap lobe lighted up as well (Supplementary Movie S2). Following the touch induced $\mathrm{Ca}^{2+}$ rise in the podium of the sender it dropped to base line levels with double exponential kinetics $\left(\operatorname{tau}_{1}=0.55 \pm 0.44 \mathrm{~s}\right.$ and $\operatorname{tau}_{2}=$ $4.3 \pm 2.02 \mathrm{~s}$ ). The calcium fluorescence faded away in the indentation zone first, a finding in agreement with the notion that cells that were activated initially are subject of bleach first. This $\mathrm{Ca}^{2+}$-signal in the sender trigger hair and two receivers moved with a velocity of $2.66 \pm$ $0.18 \mathrm{~mm} / \mathrm{s}$ (mean $\pm S D ; n=4$ ) from the podium toward the hair tip.

Fig. 1

A

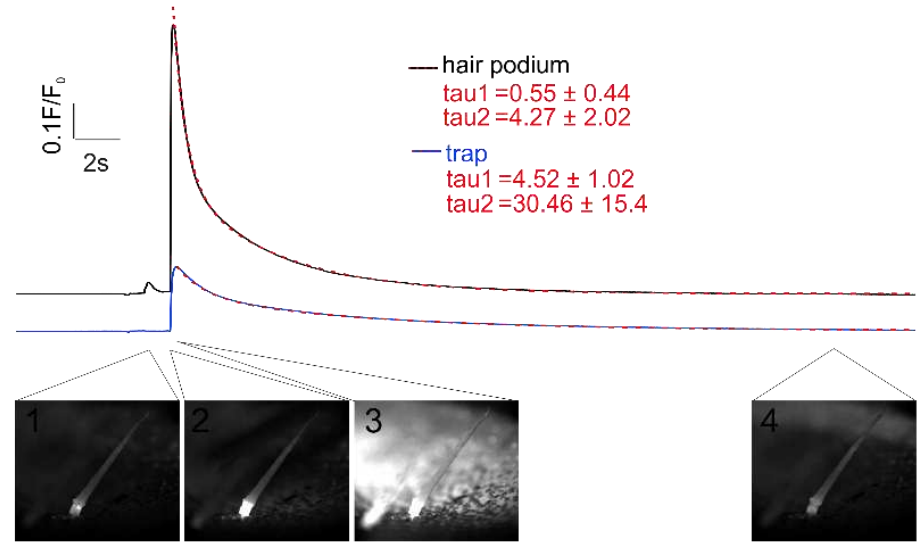

B

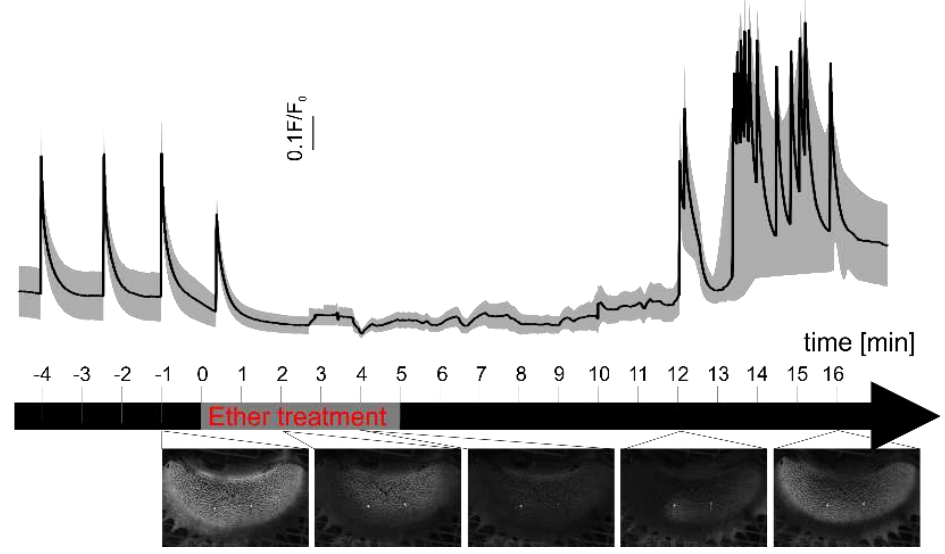

Ether inhibits hair podium [Ca] ext spread into the trap.

(A) Representative time courses of the fluorescence intensity measured in the trigger hair podium (black) and in the trap (blue) as seen in supplementary movie S1. First subthreshold stimulus only was detected in the hair (image 1). The second suprathreshold stimulation occurred first in the trap podium (image 2) and then spread to the trap tissue (image3) before returning to baseline (image 4). Decay curves of fluorescence intensities were best fitted using a double exponential function (dotted red line) with the given tau values of 4 different traps (mean $\pm S D ; n=4$ ).

(B) Timeline of the ether anesthesia of a trap. The traps were mechanically stimulated on the trigger hair every minute. At timepoint 0 , a saturated ether atmosphere was added for 5 minutes. After ventilation with fresh air, first $\mathrm{Ca}^{2+}$ signals were measured after 12 minutes again (mean \pm SD (shading); $n=4$ ). Below are sample images from the Supplementary Movie S3 of the indicated timepoints.

\section{Ether suppresses the touch induced $\mathrm{Ca}^{2+}$ wave}

To investigate the ether effect on the $\mathrm{Ca}^{2+}$ signal, one trap lobe was cut off a Dionaea leaf and fixed a microscope stage. The sender trigger hair was stimulated every 1 min with ether 
fumigation initiated after the third AP (Fig1B and Supplementary Movie S3). Already after 2 min exposed to the ether atmosphere the touch-induced $\mathrm{Ca}^{2+}$-wave did not travel the entire trap anymore but just an area around the trigger hairs. In this situation the podia of the trigger hairs still emitted a pronounced GCaMP6f fluorescence. Another 2 min later, touch triggered a Ca ${ }^{2+}$-signal in the sender trigger hair only. When compared to ether-free conditions, we did not find ether to affect the nature and amplitude of the podium $\mathrm{Ca}^{2+}$ transient much. With prolonged anesthesia this situation did not change, indication that $\mathrm{Ca}^{2+}$ channels and $\mathrm{Ca}^{2+}$ ATPases (addressed below) involved in mechano-transduction and shaping the $\mathrm{Ca}^{2+}$ transient in the trigger hair podium do not present ether targets.

While replacing the ether atmosphere by fresh air it took about 7 min to see the touch induced $\mathrm{Ca}^{2+}$ wave coming back. Initially it did not travel the full trap surface, but the area right hand side of the capture organ displayed in the video (Fig1B and Supplementary Movie S3). Only 4 min later, however, the capacity of the trigger-hair induced $\mathrm{Ca}^{2+}$ wave had reconstituted fully.

\section{Ether suppresses the touch-induced AP}

To study how ether affects the AP, we have placed a Dionaea plant in box that can be fumigated with ether. Traps were fixed to the recording chamber and a L-shaped glass capillary under the control of a micromanipulator positioned in proximity of a trigger hair. This computerized device allowed us to bend the trigger hair repeatedly and reproducibly (Supplementary Movie S4). Using surface electrodes initially, we recorded the AP in ether becoming progressively smaller until it faded away completely. In fresh air again the AP recovered its pre-stimulation shape (Fig. S2). To resolve how ether effect the AP different phases quantitatively, we inserted sharp intracellular electrodes into excitable lobe cells. In line with the literature, under control conditions the flytrap membrane potential depicted in Figure 2 was resting at about $-120 \mathrm{mV}$ (Böhm and Scherzer, 2021). Following trigger hair stimulation all-or-nothing APs got fired that under higher time resolution (insert in Fig. 2A) could be decomposed in to 6 phases (c.f. (Fabricant et al., 2021)): i) an initial fast depolarization phase to $-60 \mathrm{mV}$ which was followed by ii) a slower phase reaching the peak depolarization voltage of $-20 \mathrm{mV}$. The depolarization phase is composed of iii) an initial fast component during which the membrane voltage repolarizes to $-60 \mathrm{mV}$ and iv) an initial lower component restoring the pre-stimulation voltage of $-120 \mathrm{mV}$ that merges with an even slower v) hyperpolarization phase that peaked at $-130 \mathrm{mV}$. From this hyperpolarization overshoot the 
membrane potential in vi) final, a yet slow recovery phase returned to its pre-stimulus resting level again.

Fig. 2

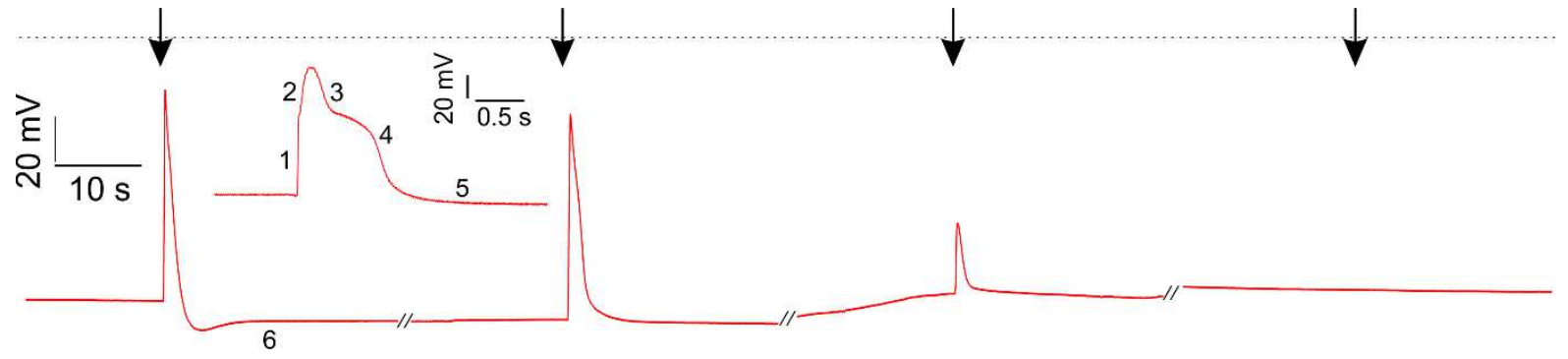

Ether inhibits electrical signal transduction in the traps.

Dry impalement of a Dionaea trap. The magnification of the first AP illustrates the individual phases in the Dionaea AP. The trap was mechanically stimulated every 2 minutes (arrows) and a saturated ether atmosphere was added after the first AP. As a result, the resting potential remained relatively constant, while the depolarisation continued to decrease until no AP could be measured in the trap after 6 minutes.

When exposed to an ether atmosphere the resting potential did not change much but 5 min after anesthesia onset the peak depolarization dropped to $-40 \mathrm{mV}$ and the overshoot hyperpolarization vanished completely. The overshoot results from a depolarization amplitude dependent transient hyperactivation of the AHA type $\mathrm{H}^{+}$ATPase (Reyer et al., 2020). The depolarization phase of the Dionaea AP results from $\mathrm{Ca}^{2+}$ influx and $\mathrm{Ca}^{2+}$ activation of the anion channel (Hedrich and Fukushima, 2021, Beilby, 1984, Beilby, 2007, Shepherd et al., 2008). In presence of the general $\mathrm{Ca}^{2+}$ channel inhibitor $\mathrm{La}^{3+}$ touch induction both action potential (Hodick and Sievers, 1988) and $\mathrm{Ca}^{2+}$ wave is suppressed (Suda et al., 2020). This indicates that both signals are interconnected and that the $\mathrm{Ca}^{2+}$ channel blocker $\mathrm{La}^{3+}$ as well as ether anesthetics is suppressing the transition of $\mathrm{Ca}^{2+}$ signal and AP from the trigger hair podium into the trap. Anesthesia, however, does not seem to target podium located mechano-sensitive $\mathrm{Ca}^{2+}$ channels and $\mathrm{Ca}^{2+}$ ATPases (addressed below).

\section{Calcium channels and pumps located in the trigger hair podium}

In losip et al. we have isolated trigger hairs and sequenced the RNAs expressed in the mechano-sensory organ (losip et al., 2020). Before trap had not opened during development it is regarded unmature and classified stage $V$ (Fig. 3A and Fig. 4). We used stage $V$ traps to compare touch/wounding calcium-electrical response. In contrast to mature traps in this developmental stage wounding did not evoke classical Dionaea APs but slow wave potentials (Fig. 3A). Upon touching trigger hairs with a fine brush, the entire unmature sensory organ 
and neighborhood co-stimulated accidentally lighted up (Fig. 3B and Supplementary Movie $\mathrm{S} 5)$. The $\mathrm{Ca}^{2+}$ signal, however, remained local rather than traveling the stage $\mathrm{V}$. This indicates that electrical and $\mathrm{Ca}^{2+}$ response exist in both stages but fast, long-distance calcium-electrical signal manifest only during maturation from stage $\mathrm{V}$ to $\mathrm{VI}$.

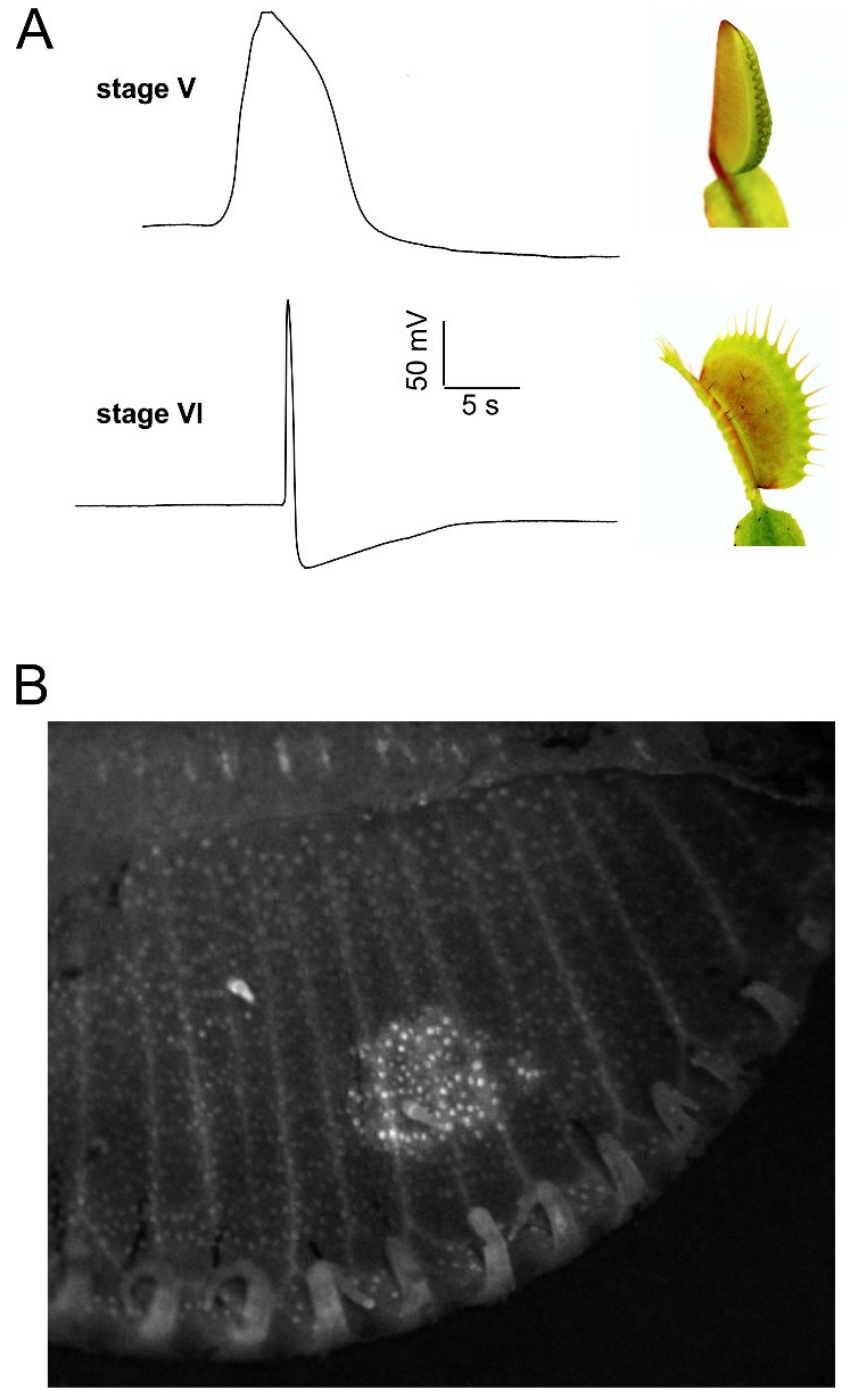

\section{Fig. 3}

Wounding induced electrochemical signal transduction in Dionaea traps

(A) Surface potential measurements of an immature stage $\mathrm{V}$ trap (top) and an adult stage $\mathrm{VI}$ trap (bottom) after wounding. Compared to the AP in adult traps, stage $V$ traps only elicit a slow wave potential upon wounding. (B) The corresponding $\mathrm{Ca}^{2+}$ signal in stage $\mathrm{V}$ GCaMP6f traps is locally limited and does not spread fast over the whole trap (example image from Supplementary Movie S5). (C) Representative time courses of the fluorescence intensity measured in the wounded stage VI trap tissue (blue) and surrounding tissue (black) (from Supplementary Movie S6). The wounding results in a fast AP (c.f. (A)) which leads to a fast $\mathrm{Ca}^{2+}$ signal in the stage VI trap. However, unlike in the undamaged tissue, the signal does not decay rapidly in the wounded tissue. Thus, only the uninjured tissue can be well fitted with the double exponential function (red).

To spot the potential ether target, we reinvestigated and extended the RNA species that have been associated with $\mathrm{Ca}^{2+}$ channels, pumps, and carriers. The

\section{C}

wounding

tau1 $=16.7 \pm 7.7$

tau2 $=$ not detectable

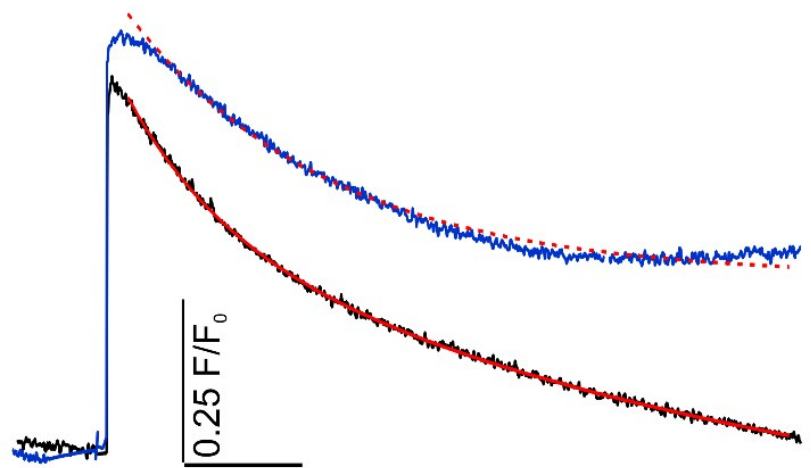

hair

tau1 $=8.2 \pm 4.4$

tau2 $=49.8 \pm 15.7$

following search criteria were used: i) genes are expressed in the trigger hair (losip et al., 2020) ii) expression is induced when trigger hairs maturate, developing trigger hair-only mechano-sensitivity and iii) genes should predominately express in the podium.

Extensive transcriptomic analyses previously revealed the expression of 
numerous transporters in the Dionaea trigger hair (losip et al., 2020), with some being specific to the trigger hair such as MSL10 and the Shaker $\mathrm{K}^{+}$channels KDM1 and DmSKOR. Moreover, the glutamate receptors DmGLR3.4 and DmGLR3.6 as well as the Ca ${ }^{2+}$-ATPase DmACA2 were characterized as trigger hair specific as well. Apart from DmGLR3.4 all of these transporters we found predominantly expressed in the podium fraction of mature trigger hair (Fig. 4, Supplementary Fig S4, and Table S1). The Ca ${ }^{2+}$-ATPase DmACA10 followed the same search criteria as well. qPCR analyses revealed that DMMSL10, KDM1, DmSKOR, DmGLR3.4, DmGLR3.6 and DmACA10 are transcriptionally induced in mature traps and fully functional stage VI trigger hairs, thus likely to contribute to the trap's calcium and electrical excitability (Fig. 4). In a previous study, an OSCA (represented by Dm_00001755-RA in (Palfalvi et al., 2020)) was described as trigger hair specific. However, the expression of this gene is rather low. We therefore re-analyzed our trigger hair transcriptome and identified another OSCA1.7 type mechanosensitive $\mathrm{Ca}^{2+}$-channel in the trigger hair which we named Dm (Dm_00005287RA) based on phylogenetic analyses. DmOSCA1.7 is expressed in the trigger hair and the mature trap and induced in both tissues during trap maturation and gain of electrical excitability (Fig. 4, Supplementary Fig S4, and Table S1).

In addition to DmOSCA1.7 we found DmGLR3.6 that represents homolog to Arabidopsis glutamate receptor channels GLR3.1/3.3 involved in long-distance mechanical stress electrical and $\mathrm{Ca}^{2+}$ signaling (Nguyen et al., 2018, Toyota et al., 2018). Loss-of-function mutants still respond to wounding locally but not systemically. In other words, in the mutant the slow wave potential (SWP; 8 times longer and 10 times slower compared to a Dionaea AP) and $\mathrm{Ca}^{2+}$ wave does not travel out of the locally wounded leaf. Furthermore, the Simon Gilroy lab has shown that upon wounding glutamate is released from injured cells (Toyota et al., 2018). When glutamate was applied to pre-wounded Arabidopsis leaves an electrical signal and a calcium wave was elicited (Toyota et al., 2018, Qi et al., 2006). 
Fig. 4
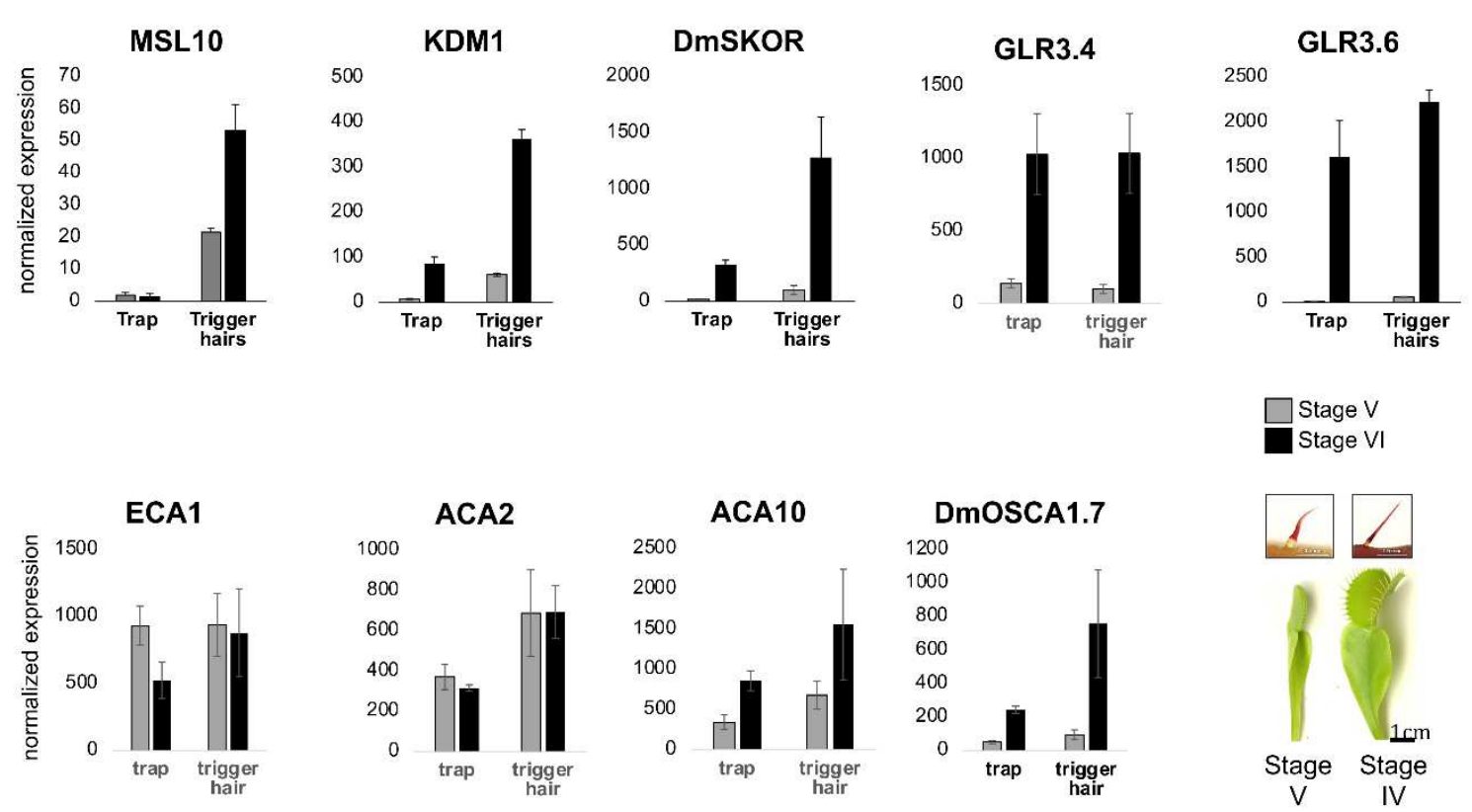

Developmental and tissue-specific expression analyses

Bottom right: Juvenile (stage $\mathrm{V}$ ) trap with the corresponding juvenile TH and adult (stage $\mathrm{VI}$ ) trap with the corresponding adult and electrically excitable $\mathrm{TH}$.

Normalized expression values of selected transporters (c.f. Supplementary Table S1) quantified by qPCR in stage $V$ and stage $\mathrm{VI}$ trap and trigger hair (mean normalized to 10,000 actin $\pm S E ; n=3-6$ ).

\section{Ether suppresses flytrap wounding and glutamate induced calcium signal transduction}

Given glutamate release and GLRs are associated with wounding, we with an Eppendorf pipette tip pinched selected sites on traps (c.f. (Scherzer et al., 2019)) of GCaMP6f expressing Dionaea and monitored the calcium-electrical response. Following injury with a macroscopic pipette tip a rapid electrical- and calcium-signal was evoked that was spreading from the compressed trap site distally. In contrast to the stimulation of the trigger hairs (Supplementary Movie S6 left), the local calcium increase induced by the compression (right) was long-lasting. One min following pinch stimulation GCaMP6f fluorescence emission had drop by $49 \%$ only (Fig. 3C and Supplementary Movie S6 right). When the trap was wounded by penetration with the pipette tip, the calcium signal in the vessels branching off from the injured section even outweighed the parenchyma signal (Supplementary Movie S7). The latter response might be explained by glutamate release in response to wound addressing GLR receptors to $\mathrm{Ca}^{2+}$ activate in the vasculature (c.f. (Toyota et al., 2018)). 
Dionaea's response to glutamate was tested by applying $5 \mathrm{mM}$ of the key amino acid in animal cells serving as neurotransmitter. To guarantee that extracellularly fed glutamate reach its targets in the trap, we cut off one trap lobe. After a wounding-recovery phase and the trap firing APs in response to trigger hair stimulation, glutamate was applied to the pre-wounded trap side (Supplementary Fig. S3). After adding the chemical stimulus to the vasculatureexposed midrib half, a fast AP and calcium wave travelled the trap (Fig 4B, Supplementary Movie S8).

To ask how ether is affecting the flytraps glutamate response, we initially stimulated traps via trigger hair bending once per minute (Supplementary Movie S9). After the third AP was fired, the trap was exposed an ether atmosphere. Five min later no trigger hair AP could be evoked. In this situation the trap was wounded. In this experimental series (Supplementary Movie S10) we analyzed GCaMP trap's glutamate $\mathrm{Ca}^{2+}$ response in fresh air side-by-side with those exposed to ether. In the absence of ether, the touch-induced $\mathrm{Ca}^{2+}$ signal originating in trigger hair podium spread over the entire trap, while under anesthesia the touch induced $\mathrm{Ca}^{2+}$ rise was restricted to the very mechano-sensory organ (c.f. Supplementary Movie S3). When a 0.1 $\mathrm{ml}$ drop of $5 \mathrm{mM}$ glutamate solution was applied in fresh air only it triggered a fast $\mathrm{Ca}^{2+}$ wave that moved from the side of midrib application all over the trap reaching epidermis cells, triggers hairs, nectaries, and very likely parenchyma cells too (Supplementary Movie S10).

This initial fast response was accompanied by a calcium wave travelling the vasculature. While the primary signals faded away the fluorescence of the latter became dominating. The more this kind of secondary $\mathrm{Ca}^{2+}$ wave propagated from the midrib towards the rim at a speed of only $0.3 \pm 0.05 \mathrm{~mm} / \mathrm{s}$. It is worth mentioning that glutamate stimulation enlightened the vascular strands so that the entire venation pattern became visible: strands were running highly parallel in from midrib along the secretory gland zone, branched and fused with their neighbors in the nectaries zone, and grow extensions towards the teeth. In contrast ether treated traps did not respond to the chemical simulation with $5 \mathrm{mM}$ glutamate. The application of $20 \mathrm{mM}$ glutamate 2 minutes later had also no effect in the anesthetized trap. Together this indicates that with anesthetized traps touch $\mathrm{Ca}^{2+}$ signal is caught in the trigger hair podium. In this situation, long-distance $\mathrm{Ca}^{2+}$ signaling cannot be rescued by substituting mechanical stimulation by glutamate excitation. 


\section{Discussion}

\section{Trigger hair podium is side of hapto-calcium-electric signaling}

We have shown that a threshold in the trigger hair displacement amplitude exists, that when passed gives rise to an AP (Scherzer et al., 2019) and a Ca ${ }^{2+}$ wave (Fig. 1A). Below this threshold the amount of 'seed $\mathrm{Ca}^{2+\text { ' }}$ entering the mechanosensitive cells of indentation zone does not auto-amplify the $\mathrm{Ca}^{2+}$ singnal. When the local $\mathrm{Ca}^{2+}$ rises above threshold, via a $\mathrm{ClCR}$ (Calcium Induced Calcium Release)-related a self-propagating $\mathrm{Ca}^{2+}$ wave, it springs up in the indentation zone, ingresses the podium, and spreads all over the trap. We have documented that the podium differentially expresses DmOSCA1.7 a homolog of the mechanosensitive $\mathrm{Ca}^{2+}$ channel AtOSCA1 (Zhai et al., 2020, Yuan et al., 2014, Thor et al., 2020). In the working model (Fig. 5) $\mathrm{Ca}^{2+}$ influx via DmOSCA1.7 is triggering CICR via a yet unknown ER Ca ${ }^{2+}$ channel. The fact that $\mathrm{Ca}^{2+}$ signal propagates fast from podium to trap but slow from the podium to the trigger tip, points a apical-basal membrane asymmetry in $\mathrm{Ca}^{2+}$ transporters. Besides the OSCA the podium specifically expresses DmGLR3.6 type $\mathrm{Ca}^{2+}$ channel, which in Arabidopsis is required for a triggered local $\mathrm{Ca}^{2+}$ signal to become systemic (Nguyen et al., 2018, Toyota et al., 2018, Mousavi et al., 2013). In the model DmGLR3.6 is the $\mathrm{Ca}^{2+}$ window to the trap. The podium is giving birth to the $\mathrm{Ca}^{2+}$ as well as the AP. The AP is initiated by the opening of a depolarizationand $\mathrm{Ca}^{2+}$ activated anion channel such as DmQUAC1 (c.f. (Imes et al., 2013, Mumm et al., 2013, Dreyer et al., 2012)). Upon mechanical activation of DmMSL10 anions are released and predepolarize the membrane. This voltage and OSCA/CICR $\mathrm{Ca}^{2+}$ above threshold activation initiated the depolarizing phase of the AP. The Shaker type voltage (depolarization) dependent GORK/SKOR type $\mathrm{K}^{+}$channel is preferentially expressed in the podium and engaged with the repolarization of the AP. For the membrane potential to recover the resting state, the QUAC1 type anion channel the cytoplasmic $\mathrm{Ca}^{2+}$ level must drop. This task is accomplished by the two podium differentially expressed plasma membrane $\mathrm{Ca}^{2+}$ ATPase DmACA2 and 10 the ER $\mathrm{Ca}^{2+}$ pump DmECA1.

\section{Ether counteracts glutamate induced calcium-electrics}

The primary site of action of general anesthetics is the nervous system, where anesthetics like ether inhibit neuronal transmission. Anesthetics directly target a subset of plasma membrane cation channels. Our studies have shown that ether does not impair touch induced $\mathrm{Ca}^{2+}$ 
transients, excluding an OSCA as target of inhibition. Ether anesthetics and the $\mathrm{Ca}^{2+}$ channel blocker La ${ }^{3+}$ (Hodick and Sievers, 1988), however, prevents the touch- and glutamate induced $\mathrm{Ca}^{2+}$ electric signal to exit the podium. A similar lack of mechanical and glutamate dependent initiation of long-distance calcium electrical signal is known from an Arabidopsis mutant that lost glr3.1/3.3. These two AtGLRs were found to express in the vasculature (Toyota et al., 2018).

The Dionaea genome encodes 22 GLRs and DmGLR3.6 is differentially expressed in the trigger hair podium. Upon glutamate application the vasculature emitted a particularly strong GCAMP6f $\mathrm{Ca}^{2+}$ fluorescence emission but not in the presence of ether. It is thus tempting to speculate that in Dionaea DmGLR3.6 in the trigger hair podium and those expressed outside the mechano-sensor represent ether targets. This working hypothesis in future studies can be tested for DmGLR3.6 functionally complementation and anesthesia of the Arabidopsis glr3.1/3.3 mutant.

Fig. 5
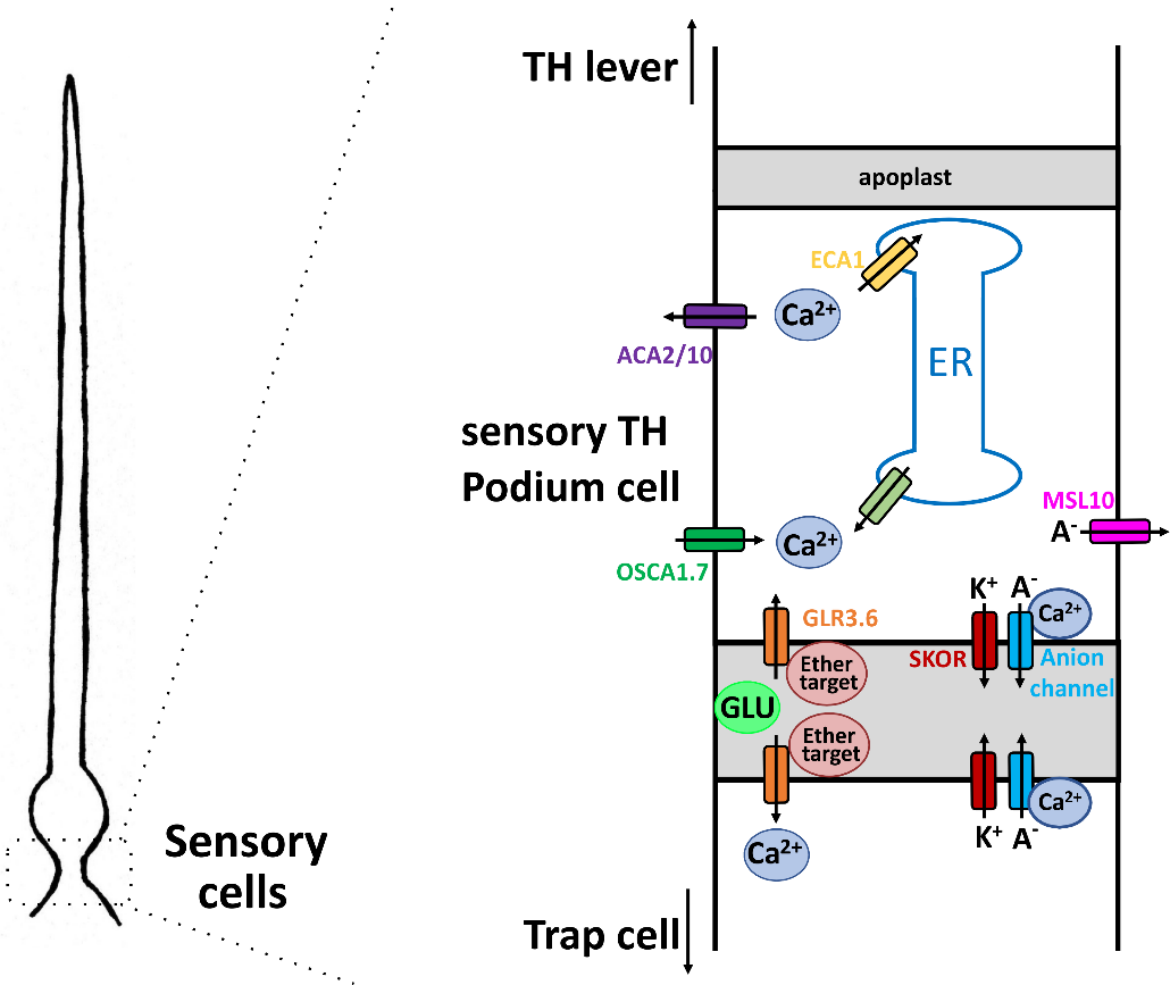

Working hypothesis on signal formation and propagation in the trigger hair.

Schematic drawing of a trigger hair (left) and the magnification of a sensory podium cell (right). Mechanical stimulation activates MSL10 (pink) and OSCA1.7 (green) which results in a depolarization an increased $\left[\mathrm{Ca}^{2+}\right]_{\mathrm{cyt}}$, which further increases via calcium induced calcium release (light green) of the endoplasmic reticulum. $\mathrm{Ca}^{2+}$-induced activation of anion channels (blue) completes the depolarisation phase of the AP, which is repolarised by activation of potassium channels (red). The resting calcium concentration is restored by the ECA1 (yellow) and ACA2/10 (purple) pumps. Apoplastic glutamate activates GLR3.6 channels (orange) and transits the $\mathrm{Ca}^{2+}$ signal into the unstimulated trap. The application of ether (red) most likely suppresses $\mathrm{Ca}^{2+}$ signalling into the trap by blocking these glutamate receptors and thus the electrical AP in the trap remains absent. 


\section{Materials and methods}

\section{Microscopy}

Stereoscopic images and videos were taken using a Leica M165 FC fluorescence stereo microscope, a Leica EL6000 lightsource, the Leica filter set ET GFP LP (Leica Microsystems, Germany), and a cooled CCD camera (Hamamatsu C4742-80-12AG, Hamamatsu Photonics, Herrsching, Germany). For GCaMP6f calcium imaging, an excitation wavelength of $475 \mathrm{~nm}$ and an exposure time of $50 \mathrm{~ms}$ at $2 \mathrm{x}$ binning was used. Fluorescence videos were taken as a sequence of uncompressed tiff files in a range where the fluorescence intensities of samples and brightness values of captured images were linear. Ether was applied by adding $2 \mathrm{ml}$ ether to a glass container next to the sample in a gas-tight setup.10x time laps for Movies S3, S8, S9 and S10 was achieved using Windows Movie Maker (Microsoft), Side by side views were produced in Premiere Pro (Adobe) and compression to MPEG2 was done in VLC media player (VideoLan). To calculate the propagation velocity of the $\left[\mathrm{Ca}^{2+}\right]_{\mathrm{cyt}}$ increase a sample rate of 25 ms was chosen. Hairs and traps were background subtracted and the mean velocity was calculated over known distances. For quantitative analysis of areas with increased $\left[\mathrm{Ca}^{2+}\right]_{\mathrm{cyt}}$ after stimulation two ROIs (regions of interest) with the same pixel numbers were defined in the indicated tissues using ImageJ. Data points were fitted using Igor Pro 8.

\section{Plant material and tissue sampling}

Dionaea muscipula GCaMP6f plants were provided from Mitsuyasu Hasebe lab (Suda et al., 2020) and grown in plastic pots at $22{ }^{\circ} \mathrm{C}$ in a $16: 8 \mathrm{~h}$ light:dark photoperiod. For microscopic experiments traps were bisected and the half which was still connected to the plant was fixed to a microscope slide the day before measurements.

For the qPCR expression measurements of genes of interest in wild-type plants, the trigger hair was dissected in two parts: the basal part and the tip. Entire/un-dissected trigger hairs were also collected as a control. For the whole trigger hairs qPCR expression analysis 300 - 600 trigger hairs were needed for one replicate, while for the tip and the base parts between 880 and 940 trigger hairs were needed to extract enough RNA for one replicate. Three replicates were used in total $n=3$. 
For qPCR analyses, RNA was isolated from each sample using the NucleoSpin Plant RNA extraction kit (Macherey-Nagel, Düren, Germany) according to the manufacturer's instructions and in combination with Fruit-mate for RNA Purification solution (Takara Bio Europe SAS, Saint-Germain-en-Laye, France). Briefly, $100 \mathrm{mg}$ of powdered plant material was thoroughly mixed with $350 \mu \mathrm{l}$ of Fruit-mate solution for 1 minute. Following 10 minutes of centrifugation $(20,000 \mathrm{rcf})$ at $4^{\circ} \mathrm{C}$, the supernatant was mixed with the lysis buffer provided by the kit (RAP), together with $3.5 \mu$ TCEP (Tris(2-carboxyethyl)phosphine hydrochloride, $0.5 \mathrm{M}, \mathrm{pH} 7$, Sigma-Aldrich). Apart from this, the kit manufacturer's instructions were followed except for the DNA digestion, which was performed in a separate step after the RNA isolation. For $1 \mu \mathrm{g}$ of RNA, $3 \mu \mathrm{l}$ DNase Buffer (10x Reaction Buffer with $\mathrm{MgCl} 2$ for DNase, Thermo Fisher Scientific, Darmstadt, Germany), $0.5 \mu$ l DNase inhibitor (RiboLock RNase Inhibitor $40 \mathrm{U} / \mu \mathrm{l}$, Thermo Fisher Scientific), and $1 \mu$ I DNase I (DNase I, RNase free 1000 units ( $1 \mathrm{U} / \mu \mathrm{l})$, Thermo Fisher Scientific) were mixed in a final volume of $30 \mu \mathrm{l}$ and incubated at $37^{\circ} \mathrm{C}$ for 30 minutes. Next, the DNA-free RNA was precipitated in isopropanol overnight at $-20^{\circ} \mathrm{C}$, together with $1 \%$ glycogen (RNA Grade (20 mg/ml), Thermo Fisher Scientific), 10\% NH4-Ac (5 mM in EDTA), 60\% Isopropanol (2-Propanol AppliChem, Darmstadt, Germany), and water up to a final volume of $100 \mu \mathrm{l}$. The samples were washed using $70 \% \mathrm{Et}-\mathrm{OH}$, centrifuged at $4^{\circ} \mathrm{C}$ for 20 minutes, and the resulting pellet was dried at $37^{\circ} \mathrm{C}$ and resuspended in water (DEPC, AppliChem).

The RNA was transcribed into cDNA using the M-MLV Reverse Transcriptase (RNase H- Point Mutant, Promega, Walldorf, Germany). qPCR was performed using a Realplex Mastercycler system (Eppendorf, Hamburg, Germany) and ABsolute QPCR SYBR green capillary mix (Thermo Fisher Scientific). Quantification of the actin transcript DmACT1 (GenBank: KC285589, Dm_00017292-RA) and transcripts for D. muscipula genes of interest was performed by realtime PCR. D. muscipula transcripts were normalized to 10,000 molecules of DmACT1.

\section{Membrane potential measurements}

Before the dry-impalement experiments, a trap was cut in half and the half that was still connected to the plant was fixed in a Petri dish on a chlorinated silver wire as a reference electrode with double-sided adhesive tape. After a 30-minute recovery, the trap was inserted with a sharp impalement electrode. For impalements, microelectrodes from borosilicate glass capillaries with filament (Hilgenberg,) were pulled on a horizontal laser puller (P2000, Sutter 
Instruments) and filled with $300 \mathrm{mM} \mathrm{KCl}$ and connected via an $\mathrm{Ag} / \mathrm{AgCl}$ half-cell to a headstage (1 G $\Omega, H S-2 A$, Axon Instruments). An IPA-2 amplifier (Applicable Electronics) was used, and the cells were impaled by an electronic micromanipulator (NC-30, Kleindiek Nanotechnik). To anaesthetise the trap in Figure 1, $1 \mathrm{ml}$ of ether was placed in a glass vial next to the trap and the trap was continuously stimulated at the trigger hair at 2-minute intervals until no more AP could be recorded.

To test the glutamate effect on the trap, $20 \mu \mathrm{l}$ of $5 \mathrm{mM}$ sodium L-glutamate was pipetted either onto the trap surface or onto the cut edge.

For surface potential measurements in Fig S2 and Supplementary Movie S4, we measured the extracellular potential of the trap tissue. One silver electrode was impaled into the trap surface. The reference electrode was put into the wet soil or the petiole. Electrical signals were amplified 100x and recorded with PatchMaster software (HEKA). Trigger hair stimulation or wounding to the trap tissue was applied at the given timepoints by a motorized arm.

\section{Funding information}

This work was funded in part by the German Research Foundation (DFG) Reinhart Koselleck grant 415282803 to Rainer Hedrich, and the King Saud University's International Cooperation and Scientific Twinning Dept., Riyadh, Saudi Arabia (Project ICSTD-2020/2), the Deutsche Forschungsgemeinschaft (DFG) FOR 3004 SYNABS P1, and by the Leading Initiative for Excellent Young Researchers of MEXT, Japan to K.Y..

\section{Acknowledgements}

We thank Tracey Ann Cuin for her helpful discussions and assistance in preparing this paper. We would like to thank Christian Wegener (Professor of Neurogenetics Biocenter Würzburg) for his kind support and for making his setups available. 


\section{Contributions}

S.S and R.H. proposed to study ether influence in Dionaea's signal transduction. S.S., A.I and K.Y. conducted experiments. S.S. and I.F. analysed data. R.H. and S.S. wrote the manuscript. S.H., K.A.S.R., and M.H.R.H. edited the manuscript.

\section{Declarations}

The authors declare no competing interests and follow relevant guidelines and legislation. 


\section{$\underline{\text { References }}$}

ALFIERI, A., DOCCULA, F. G., PEDERZOLI, R., GRENZI, M., BONZA, M. C., LUONI, L., CANDEO, A., ROMANO ARMADA, N., BARBIROLI, A., VALENTINI, G., SCHNEIDER, T. R., BASSI, A., BOLOGNESI, M., NARDINI, M. \& COSTA, A. 2020. The structural bases for agonist diversity in an Arabidopsis thaliana glutamate receptor-like channel. Proc Natl Acad Sci U S A, 117, 752-760.

BEILBY, M. J. 1984. Calcium and Plant Action-Potentials. Plant Cell and Environment, 7, 415421.

BEILBY, M. J. 2007. Action potential in charophytes. International Review of Cytology - a Survey of Cell Biology, Vol 257, 257, 43-82.

BÖHM, J. \& SCHERZER, S. 2021. Signalling and transport processes related to the carnivorous lifestyle of plants living on nutrient-poor soil. Plant Physiology.

BOHM, J., SCHERZER, S., KROL, E., KREUZER, I., VON MEYER, K., LOREY, C., MUELLER, T. D., SHABALA, L., MONTE, I., SOLANO, R., AL-RASHEID, K. A., RENNENBERG, H., SHABALA, S., NEHER, E. \& HEDRICH, R. 2016a. The Venus Flytrap Dionaea muscipula Counts Prey-Induced Action Potentials to Induce Sodium Uptake. Curr Biol, 26, 286-95.

BOHM, J., SCHERZER, S., SHABALA, S., KROL, E., NEHER, E., MUELLER, T. D. \& HEDRICH, R. 2016b. Venus Flytrap HKT1-Type Channel Provides for Prey Sodium Uptake into Carnivorous Plant Without Conflicting with Electrical Excitability. Mol Plant, 9, 428436.

BROSNAN, R. J. \& PHAM, T. L. 2018. Anesthetic-sensitive ion channel modulation is associated with a molar water solubility cut-off. BMC Pharmacol Toxicol, 19, 57.

COVARRUBIAS, M., BARBER, A. F., CARNEVALE, V., TREPTOW, W. \& ECKENHOFF, R. G. 2015. Mechanistic Insights into the Modulation of Voltage-Gated Ion Channels by Inhalational Anesthetics. Biophys J, 109, 2003-11.

DREYER, I., GOMEZ-PORRAS, J. L., RIANO-PACHON, D. M., HEDRICH, R. \& GEIGER, D. 2012. Molecular Evolution of Slow and Quick Anion Channels (SLACs and QUACs/ALMTs). Front Plant Sci, 3, 263.

FABRICANT, A., IWATA, G. Z., SCHERZER, S., BOUGAS, L., ROLFS, K., JODKO-WLADZINSKA, A., VOIGT, J., HEDRICH, R. \& BUDKER, D. 2021. Action potentials induce biomagnetic fields in carnivorous Venus flytrap plants. Sci Rep, 11, 1438.

GANGWAR, S. P., GREEN, M. N., MICHARD, E., SIMON, A. A., FEIJO, J. A. \& SOBOLEVSKY, A. I. 2020. Structure of the Arabidopsis Glutamate Receptor-like Channel GLR3.2 LigandBinding Domain. Structure.

HEDRICH, R. \& FUKUSHIMA, K. 2021. On the Origin of Carnivory: Molecular Physiology and Evolution of Plants on an Animal Diet. Annu Rev Plant Biol, 72, 133-153.

HEDRICH, R. \& NEHER, E. 2018. Venus Flytrap: How an Excitable, Carnivorous Plant Works. Trends Plant Sci, 23, 220-234.

HODICK, D. \& SIEVERS, A. 1988. The Action-Potential of Dionaea-Muscipula Ellis. Planta, 174, 8-18.

IMES, D., MUMM, P., BOHM, J., AL-RASHEID, K. A., MARTEN, I., GEIGER, D. \& HEDRICH, R. 2013. Open stomata 1 (OST1) kinase controls R-type anion channel QUAC1 in Arabidopsis guard cells. Plant J, 74, 372-82.

IOSIP, A. L., BOHM, J., SCHERZER, S., AL-RASHEID, K. A. S., DREYER, I., SCHULTZ, J., BECKER, D., KREUZER, I. \& HEDRICH, R. 2020. The Venus flytrap trigger hair-specific potassium 
channel KDM1 can reestablish the $\mathrm{K}+$ gradient required for hapto-electric signaling. PLOS Biol, 18, e3000964.

MAKSAEV, G. \& HASWELL, E. S. 2012. MscS-Like10 is a stretch-activated ion channel from Arabidopsis thaliana with a preference for anions. Proc Natl Acad Sci U S A, 109, 19015-20.

MOUSAVI, S. A., CHAUVIN, A., PASCAUD, F., KELLENBERGER, S. \& FARMER, E. E. 2013. GLUTAMATE RECEPTOR-LIKE genes mediate leaf-to-leaf wound signalling. Nature, 500, 422-6.

MUMM, P., IMES, D., MARTINOIA, E., AL-RASHEID, K. A., GEIGER, D., MARTEN, I. \& HEDRICH, R. 2013. C-terminus-mediated voltage gating of Arabidopsis guard cell anion channel QUAC1. Mol Plant, 6, 1550-63.

MURTHY, S. E., DUBIN, A. E., WHITWAM, T., JOJOA-CRUZ, S., CAHALAN, S. M., MOUSAVI, S. A. R., WARD, A. B. \& PATAPOUTIAN, A. 2018. OSCA/TMEM63 are an Evolutionarily Conserved Family of Mechanically Activated Ion Channels. Elife, 7.

NGUYEN, C. T., KURENDA, A., STOLZ, S., CHETELAT, A. \& FARMER, E. E. 2018. Identification of cell populations necessary for leaf-to-leaf electrical signaling in a wounded plant. Proceedings of the National Academy of Sciences of the United States of America, 115, 10178-10183.

PALFALVI, G., HACKL, T., TERHOEVEN, N., SHIBATA, T. F., NISHIYAMA, T., ANKENBRAND, M., BECKER, D., FORSTER, F., FREUND, M., IOSIP, A., KREUZER, I., SAUL, F., KAMIDA, C., FUKUSHIMA, K., SHIGENOBU, S., TAMADA, Y., ADAMEC, L., HOSHI, Y., UEDA, K., WINKELMANN, T., FUCHS, J., SCHUBERT, I., SCHWACKE, R., AL-RASHEID, K., SCHULTZ, J., HASEBE, M. \& HEDRICH, R. 2020. Genomes of the Venus Flytrap and Close Relatives Unveil the Roots of Plant Carnivory. Curr Biol, 30, 2312-2320 e5.

PAVEL, M. A., PETERSEN, E. N., WANG, H., LERNER, R. A. \& HANSEN, S. B. 2020. Studies on the mechanism of general anesthesia. Proc Natl Acad Sci U S A, 117, 13757-13766.

PAVLOVIC, A., LIBIAKOVA, M., BOKOR, B., JAKSOVA, J., PETRIK, I., NOVAK, O. \& BALUSKA, F. 2020. Anaesthesia with diethyl ether impairs jasmonate signalling in the carnivorous plant Venus flytrap (Dionaea muscipula). Ann Bot, 125, 173-183.

PROCKO, C., MURTHY, S., KEENAN, W. T., MOUSAVI, S. A. R., DABI, T., COOMBS, A., PROCKO, E., BAIRD, L., PATAPOUTIAN, A. \& CHORY, J. 2021. Stretch-activated ion channels identified in the touch-sensitive structures of carnivorous Droseraceae plants. Elife, 10.

QI, Z., STEPHENS, N. R. \& SPALDING, E. P. 2006. Calcium entry mediated by GLR3.3, an Arabidopsis glutamate receptor with a broad agonist profile. Plant Physiol, 142, 96371.

REYER, A., HASSLER, M., SCHERZER, S., HUANG, S., PEDERSEN, J. T., AL-RASCHEID, K. A. S., BAMBERG, E., PALMGREN, M., DREYER, I., NAGEL, G., HEDRICH, R. \& BECKER, D. 2020. Channelrhodopsin-mediated optogenetics highlights a central role of depolarization-dependent plant proton pumps. Proc Natl Acad Sci U S A, 117, 2092020925.

SCHERZER, S., FEDERLE, W., AL-RASHEID, K. A. S. \& HEDRICH, R. 2019. Venus flytrap trigger hairs are micronewton mechano-sensors that can detect small insect prey. Nature Plants, 5, 670-675.

SHEPHERD, V. A., BEILBY, M. J., AL KHAZAALY, S. A. S. \& SHIMMEN, T. 2008. Mechanoperception in Chara cells: the influence of salinity and calcium on touch-activated receptor potentials, action potentials and ion transport. Plant Cell and Environment, $31,1575-1591$. 
SUDA, H., MANO, H., TOYOTA, M., FUKUSHIMA, K., MIMURA, T., TSUTSUI, I., HEDRICH, R., TAMADA, Y. \& HASEBE, M. 2020. Calcium dynamics during trap closure visualized in transgenic Venus flytrap. Nature Plants.

THOR, K., JIANG, S., MICHARD, E., GEORGE, J., SCHERZER, S., HUANG, S., DINDAS, J., DERBYSHIRE, P., LEITÃO, N., DEFALCO, T. A., KÖSTER, P., HUNTER, K., KIMURA, S., GRONNIER, J., STRANSFELD, L., KADOTA, Y., BÜCHERL, C. A., CHARPENTIER, M., WRZACZEK, M., MACLEAN, D., OLDROYD, G. E. D., MENKE, F. L. H., ROELFSEMA, M. R. G., HEDRICH, R., FEIJÓ, J. \& ZIPFEL, C. 2020. The calcium-permeable channel OSCA1.3 regulates plant stomatal immunity. Nature.

TOYOTA, M., SPENCER, D., SAWAI-TOYOTA, S., WANG, J. Q., ZHANG, T., KOO, A. J., HOWE, G. A. \& GILROY, S. 2018. Glutamate triggers long-distance, calcium-based plant defense signaling. Science, 361, 1112-+.

YUAN, F., YANG, H., XUE, Y., KONG, D., YE, R., LI, C., ZHANG, J., THEPRUNGSIRIKUL, L., SHRIFT, T., KRICHILSKY, B., JOHNSON, D. M., SWIFT, G. B., HE, Y., SIEDOW, J. N. \& PEI, Z. M. 2014. OSCA1 mediates osmotic-stress-evoked Ca2+ increases vital for osmosensing in Arabidopsis. Nature, 514, 367-71.

ZHAI, Y., WEN, Z., HAN, Y., ZHUO, W., WANG, F., XI, C., LIU, J., GAO, P., ZHAO, H., WANG, Y., WANG, Y. \& HAN, S. 2020. Heterogeneous expression of plasma-membrane-localised OsOSCA1.4 complements osmotic sensing based on hyperosmolality and salt stress in Arabidopsis osca1 mutant. Cell Calcium, 91, 102261. 


\section{Supplementary Files}

This is a list of supplementary files associated with this preprint. Click to download.

- SupplementaryMovieS1.ts

- SupplementaryMovieS1010x.ts

- SupplementaryMovieS2.ts

- SupplementaryMovieS310x.ts

- SupplementaryMovieS4.ts

- SupplementaryMovieS5.ts

- SupplementaryMovieS6.ts

- SupplementaryMovieS7.ts

- SupplementaryMovieS810x.ts

- SupplementaryMovieS910x.ts

- Supplementaryinformation.pdf 